\title{
Editorial
}

\section{Brittle or Quasi-Brittle Fracture of Engineering Materials 2016}

\author{
Filippo Berto, ${ }^{1}$ Liviu Marsavina, ${ }^{2}$ Majid R. Ayatollahi, ${ }^{3}$ \\ Sergei V. Panin, ${ }^{4}$ and Konstantinos I. Tserpes ${ }^{5}$ \\ ${ }^{1}$ Department of Engineering Design and Materials, Norwegian University of Science and Technology (NTNU), \\ 7491 Trondheim, Norway \\ ${ }^{2}$ Faculty of Mechanical Engineering, Politehnica University of Timisoara, Blvd. M. Viteazu, Nr. 1, \\ 300222 Timisoara, Romania \\ ${ }^{3}$ Fatigue and Fracture Research Laboratory, Center of Excellence in Experimental Solid Mechanics and Dynamics, \\ School of Mechanical Engineering, Iran University of Science and Technology, Narmak, Tehran 16846, Iran \\ ${ }^{4}$ Institute of Strength Physics and Material Science, Department of Mechanical Engineering \& Aeronautics, \\ Russian Academy of Sciences, Siberian Branch, Tomsk 634021, Russia \\ ${ }^{5}$ Laboratory of Technology \& Strength of Materials (LTSM), Department of Mechanical Engineering \& Aeronautics, \\ University of Patras, 26500 Patras, Greece
}

Correspondence should be addressed to Filippo Berto; berto@gest.unipd.it

Received 22 September 2016; Accepted 22 September 2016

Copyright (c) 2016 Filippo Berto et al. This is an open access article distributed under the Creative Commons Attribution License, which permits unrestricted use, distribution, and reproduction in any medium, provided the original work is properly cited.

Brittle or quasi-brittle fracture of engineering materials is a wide field of research, which involves many researchers devoted to investigate different aspects of the mechanics and physics of fracture. Materials usually treated include metal alloys, polymers, composites, rocks, and ceramics.

Brittle failure is not a phenomenon limited only to static loading. It may also be related to the fatigue and failure under repeated loading cycles (mechanical or thermal). The material damage process is usually very complex because it involves the combined effects of loading, size and geometry, temperature, and environment. The understanding of the phenomena tied to the dissipation of energy in various forms and the identification of microscopic properties and their interactions with macroscopic variables are the actual challenging topics. The fracture mechanics science emphasizes material characterization techniques and translation of specimen data to design.

The present special issue contains original research and review articles that seek to define possible criteria against brittle and quasi-brittle failure and to present or discuss new sets of experimental data in combination with fracture assessment. Among the areas emphasized in the SI are case histories; material selection and structure design; sample calculations of practical design problems; material characterization procedures; fatigue crack growth and corrosion; nondestructive testing and inspection; structural failure and ageing; failure prevention methodologies; and maintenance and repair. The papers submitted by the authors have been subjected to the normal journal peer-review process.

The papers submitted by the authors have been subjected to the normal journal peer-review process. Special topics include but are not limited to continuum mechanics, crack propagation, criteria for fatigue and fracture assessment, micromechanics, nanomechanics, energy absorption and dissipation, local approaches based on strain energy density, local approaches based on stress analysis, scale effect, singular stress field, interface behavior of small and large bodies, and three-dimensional effects.

\section{Acknowledgments}

We thank the many anonymous reviewers who assisted us in the review process providing useful comments and proposing constructive improvements to the authors. Without their continuous and valuable support also our efforts could have been useless. 
Filippo Berto Liviu Marsavina Majid R. Ayatollahi Sergei V. Panin Konstantinos I. Tserpes 

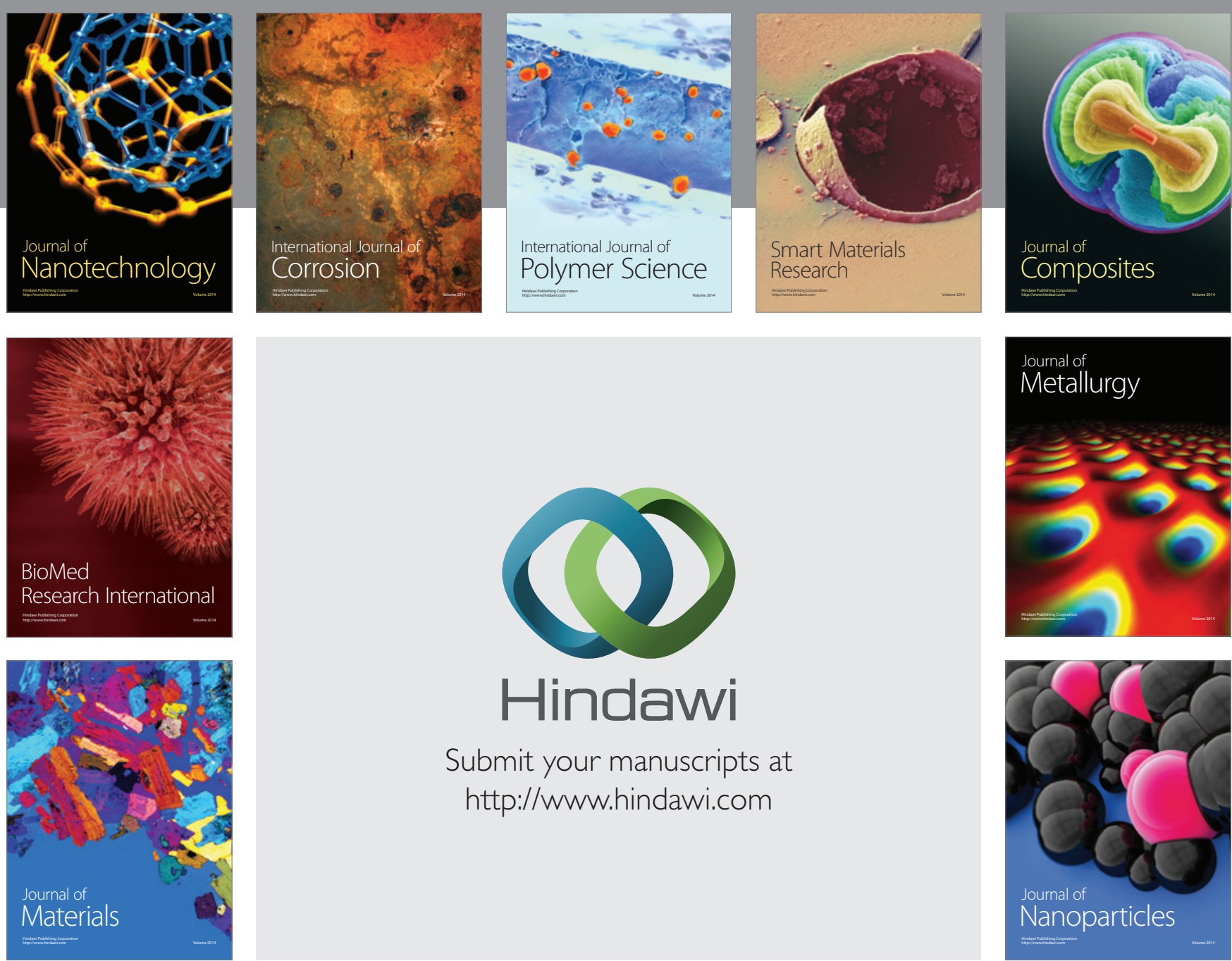

\section{Hindawi}

Submit your manuscripts at

http://www.hindawi.com

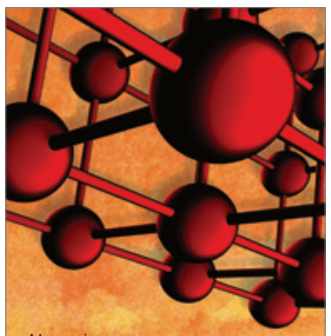

Materials Science and Engineering
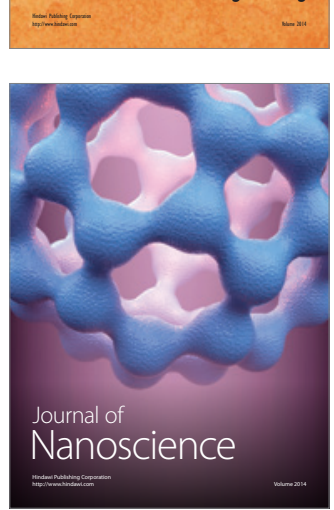
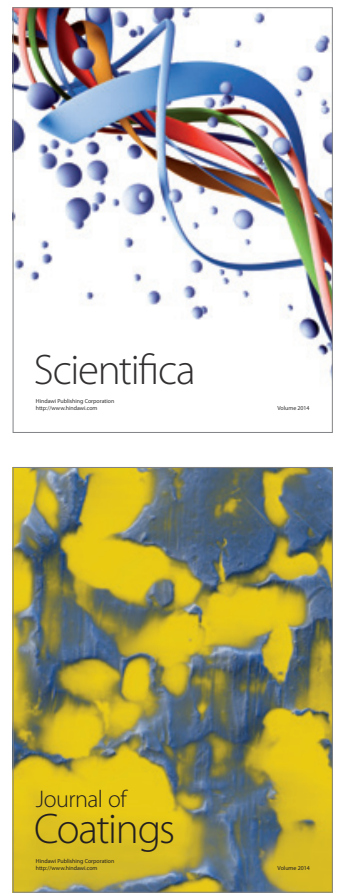
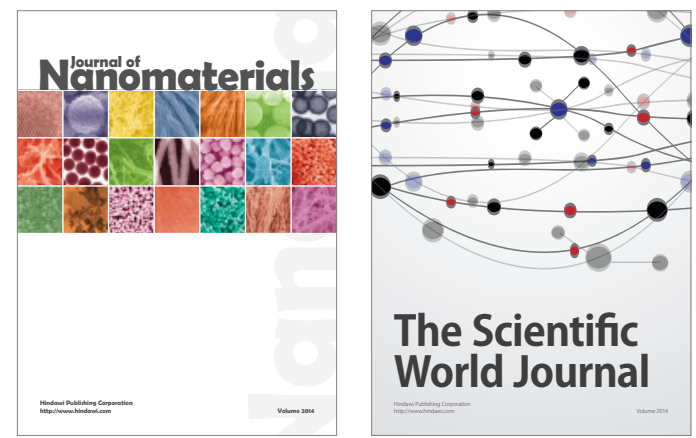

The Scientific World Journal
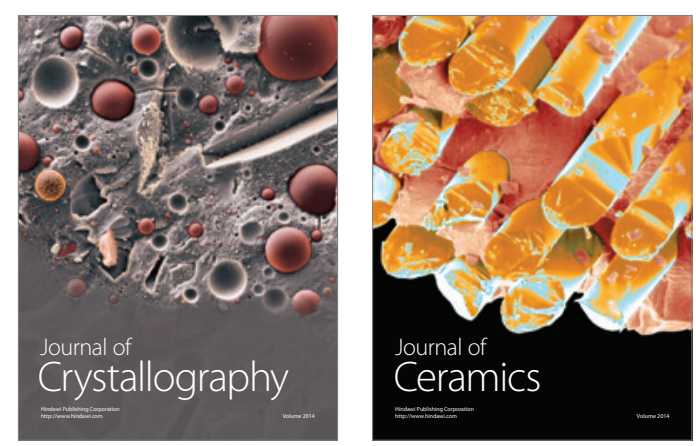
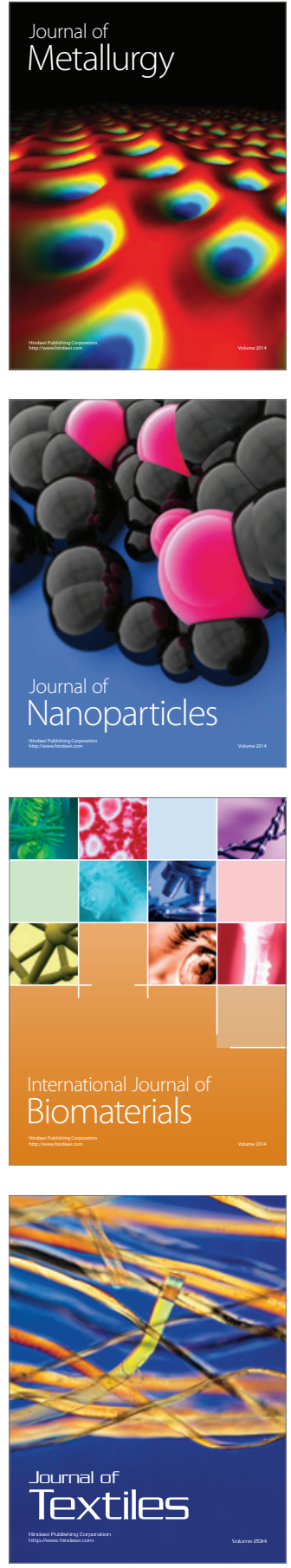